port (Fig. $2 a$ ) which can he raised or lowered to suit the patient's convenience, it being supported by a $\operatorname{cog}$ and rachet arrangement, which is also found attached to the head windlass of the bed proper (Fig $1 d)$.

At the head of the tank (Fig. $1 a$ ), but separate and distinct from same, is a water receiver (Fig. 3 ) connected to the tank by a single pipe; merging into this receiver is both a cold and hot water pipe, coming either from a range or boiler, according to the source of the hot water. The temperature of the water is hence regulated here instead of at the main tank. Naturally, the large tank (Fig. $1 a$ ) is supplied with an escape pipe and a safety escape to prevent an overflow. (Fig. $4 a, b$.)

Fig. $1 \mathrm{E}$ represents the side woodwork of the bed.

Upon the patient's arrival, orders are given the nurse to prepare the water bed, whose arrangement as you have seen is so simple that an inexperienced hand can prepare it in five minutes. The tempera. ture of the water should be about 25 or 26 degrees $R$., and increased after the immersion to 32 or 34 degrees $\mathrm{R}$.

The patient is carefully lifted onto to the blanket covering the mattress, which is swung above the water's surface, which should reach to almost onethird of the top edge of the tank. Almost at the instant the patient is immersed his heartrending shrieks and moans will subside, and if asked after five minutes, regarding his condition, will state that he now feels comfortable. If necessary, stimulants can be given and if the patient be laboring under intense shock, the water can be made somewhat warmer than that which would give the most comfortable feeling to the patient, being gauged by the hand and thermometer. To the face is generally applied a mask of linen, saturated in carron oil. Within an hour or two, after the more acute symp. toms have subsided, the patient is raised out of the water, catheterized, and the loose shreds of epidermis trimmed off and existing bullæ are tapped, care being taken not to remove the epidermial covering. Then the case is treated symptomatically.

As I have before stated, the object of these few lines is not so much the recommendation of the agent (as water has been used from time immemorial for the treatment of burns by both the laity and physi cians) as it is the method of application, and consequently I would suggest the adoption of the water bed in all hospitals; possibly some ingenious mem. ber of the profession could suggest a plan by which the idea could be carried out in private practice, namely an apparatus adjustable to any bath tub.

I am certain that, had we been possessed of such th an apparatus during my term of service at the Michae! Reese Hospital, it would have been decidedly to the patients' advantage as well as our own.

The advantages claimed for the water bed are:

1. Relieving the patient almost instantly of the excruciating pain consequent on a severe burn, and following the application and removal of dressinge afterward.

z. Producing more rapid cicatrization.

3. Relieving shock.

4. Preventing infection from erysipelas, pus microorganisms, etc.

5. Prevention of amyloid degenerations due to the confinement of secretions by dressings.

6. Saving of expense of material and time in frequent dressing.
Patients may be allowed to follow this aquatic life for months and months, Kaposi reporting one patient having spent 385 consecutive days and nights in the water bed. They eat, drink, sleep and frequently gain considerably in weight in the bed. Not alone can burns be treated in this manner, but all forms of discase in which there is an extensive loss of epidermis as in pemphigus foliaceus, gangrene, or in chronic suppurating wounds.

\section{FACIAL BLEMISHES ; A PLEA FOR THE STUDY OF THE SCIENCE AND ART OF COSMETICS.}

BY EDWARD H. SCHAEFER, M.D., Ph.L.

KANSAS CITY, MO.

There are many affections and defects of the body that demand the attention of the physician, who, in applying his knowledge of how to relieve and accomplish a cure, thinks he has done all that he is able to do.

There is still one branch left, belonging to the external part of the human frame, to which very little attention has been devoted and even arbitrarily excluded from the province of medicine, and that is the science of cosmetics. Physicians have always searched for hidden troubles of the human economy, diseases that are internal, not discernible by the eye, thereby entirely ignoring the outward conditions of man.

That branch of medicine, intimately connected with dermatology, the science and art of cosmetics, has been until recently a kind of terra incognita, being exclusively in the hands of empyrics or nonprofessional persons. For ages, men, and women especially, have striven to enhance their personal attractiveness, augmenting beauty and symmetry to their forms. What remedial means and agents could be utilized in order to beautify face and body were the source of greatest speculation. Every one, man and woman, wishes to appear attractive, which naturally is as it should be, and all means that are employed in that direction to accomplish the desired end, are truly commendable. How often will a woman refuse to take medicine for some slight trouble, but can be persuaded with ease to do so, if she is reminded that some cosmetic blemishes might be the result. The impressions made upon us by persons depend largely upon their external appearance. Anything pleasing in its nature will be estimated in a favorable measure of opinion.

Our ideas relate more or less to esthetics, be it in the form of objects or persons. Women, knowing this perquisite to attractiveness have, since the remotest ages used all such remedies at their command that would heighten their bodily charms. We all are aware how concerned women often are about any eruption on the face, as for instance, acne, lenticular spots, chloasma and other facial blemishes too numerous to mention. Should this not in itself he a mild reminder of the necessity that the physician ought to concern himself more about those diseases of the skin that occur mostly upon the face and which often leave marks or imprints? How well could he apply his knowledge of the anatomy of the skin in making operations upon the face for tumors and other growths, so as to avoid leaving cicatrices. Young men and women may be seen walking along the streets, who have their faces literally disfigured 
by acne, which often leaves its variola-like scars. If any ore takes the trouble of observing he can see numerous people who have nævi, warts and pigmen. tations, such as freckles, chloasmata and port-wine marks on their faces and necks. How often do we not notice people whose faces are disfigured by scars, due to wounds that have suppurated a long time ago and have healed by granulation? Does it not appear to every thoughtful physician that there is still a wide field open to him in facial dermatology, if he only would try to do something in the line of removing or concealing facial blemishes by cosmetics? Wrinkles, the companion of approaching age, ought to be amenable to treatment, more so if they occur in the young.

Crimivals often resort to the skill of surgeons in having certain marks removed from their faces in such a manner that not a vestige of former stigmata remains.

Among the plastic operations for the relief of harelip, cleft-palate, the operation of rhinoplasty, we realize the aim of surgery to establish the art of cosmetics. All those remedial procedures, that are employed in the form of hot or cold baths, in order to incite perspiration, as well as massage and gymnastics, are used for the sole purpose of adding beauty to the skin and complexion.

We have so many agents at our disposal that could be employed in removing all disfiguring marks of the face and other exposed portions of the body, as for instance, electrolysis, excision with the knife, skin. grafting, the application of acids and alkalies and many other methods that the discretion and inven. tiveness of the physician may contrive.

There is one most prevalent cutaneous disease which appears so often upon the faces of young people of both sexes. I refer to acne with accompanying comedones. This skin disease is curable and ought to be successfully treated in a few weeks if the attention be given to it as it deserves.

Another sub-specialty, advertised by non-professionals, namely "capillurgy," the art of destroying superfluous hair, should be practiced only by physicians.

In the field of facial dermatology very much can be improved upon, in view of the fact, that by apply ing ourselves to that branch assiduously and studying the true pathologic conditions found present in cutaneous diseases, we are enabled to treat them more intelligently and effect a cure in many cases.

\section{BLOOD-SERUM THERAPY IN DIPHTHERIA}

Read before the Chicago Medical Society, Dec. 3, 1894.

\section{BY G. E. KRIEGER, M.D.}

SURGEON TO THE CHICAGO HOSPITAL.

The blood-serum therapy of Behring, Roux and others is based upon the fundamental fact, that the blood of an animal which has been rendered immune against a certain disease has the power, when injected into another individual of the same or other species, to immunize the latter also against that disease. Suppose, for instance, we have a guinea pig immunized against tetanus or diphtheria bacilli and injected some of its blood into a rabbit, the latter would be protected for a certain length of time against infection of the germs or their poisonous products. Such products are especially dangerous and deleterious to the organism in diphtheria. If one intends to immunize an animal against the effect of diphtheria bacilli it will be necessary to increase its resistance against the toxic products of the germs, the toxins.

An increased resistance and finally an immunity is obtained by applying systematically such doses of the poison to the animal as are sufficient to make the latter ill without causing its death. As the tolerance of the animal increases, so does the immunizing power of the blood-serum increase, which has been proven by Ehrlich in reference to the vegetable poisons, and by Behring with bacterial toxins. The way of proceeding for the purpose of immunizing animals against diphtheria as advised by Behring, Ehrlich and Wasserman is hy hypodermic injections of at first very small, and later, gradually increasiug doses of diphtheria cultures and diphtheria toxins.

The latter, according to Roux and Yersin, is prepared by inoculation of beef-tea in large flasks with diphtheria bacilli. After the flasks have remained in an incubator for three to four weeks 0.5 per cent. carbolic acid or 0.33 per cent. trikresol is added, by which the germs are killed and precipitated to the bottom of the flasks. The liquid then contains the toxin in solution. From this solution the toxin can be prepared as a dry substance or it can be used while dissolved in the fluid. The effect of injections of the toxin, is fever, local swelling and the production of antitoxin. The latter is found in the blood of the animal after each injection and increases in quantity with the number of injections. This treatment is continued until the animal possesses a sufficient quantity of antitoxin. In order to estimate its efficacy, it is necessary to tap the animal, from time to time, for a small amount of blood with which experiments are made on others. Behring and Kitasato were the first who observed that such antitoxin is able to neutralize the effect of toxin, when mixed in a tube.

The mode of valuation of the diphtheria antitoxin, as advised by Ehrlich is this; of a toxin, the efficacy of which upon a guinea pig is known, a certain quantity ten times the minimum fatal dose, which may be 1 gramme is mixed with a number of different quantities of the blond to be examined, for instance $0.5,0.25,0.15,0.10 \mathrm{gr}$., and with these four mixtures four guinea pigs are inoculated. The result will be that the one which received the largest dose of the blood together with the toxin shows no reaction; the one that received the second largest dose will suffer from an acute local inflammation, followed by necrosis, but otherwise be not much affected. The third will prohably become quite sick and the one which got the smallest quantity of blood will die in forty-eight hours, from the same dose of toxin as the other received. Hereby we can find out which amount of blood of the first animal is sufficient to neutralize a certain quantity of toxin, and so the value of serum can be established. This is simplified if one has a material of a standard strength with which to compare others. Behring and Ehrlich therefore, prepared a serum of which $0.1 \mathrm{gr}$. is sufficient to neutralize ten times the fatal dose of toxin (for guinea pigs ) and this they called"normal therapeutic serum." Of such normal serum one cubic centimeter was called the equivalent for one immunizing unit. According hereto a serum, of which $0.01 \mathrm{gr}$. is sufficient to neutralize ten times the fatal dose, would possess 